\title{
Expert Discussion: Breast Cancer in Low-Resource Countries
}

\author{
Eva Johanna Kantelhardt ${ }^{a, b}$ Mathewos Assefa ${ }^{c}$ Valerie McCormack ${ }^{d}$ \\ Herbert Cubasch ${ }^{\mathrm{e}}$ Ahmedin Jemal $^{\mathrm{f}}$ Lydia E. Pace ${ }^{\mathrm{g}} \mathrm{h}$

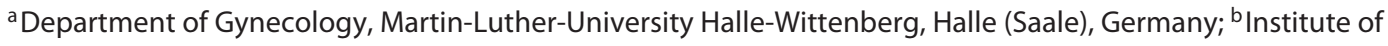 \\ Medical Epidemiology, Biostatistics and Informatics, Martin-Luther-University Halle-Wittenberg, Halle (Saale), \\ Germany; ${ }^{C}$ Radiotherapy Center, Addis Ababa University, Addis Ababa, Ethiopia; ${ }^{\mathrm{d}}$ International Agency for

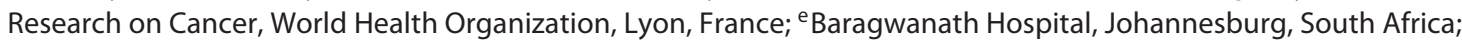 \\ f Surveillance and Health Services Research, American Cancer Society, Atlanta, GA, USA; 'Brigham and Women's \\ Hospital, Boston, MA, USA; ${ }^{\text {h}}$ Harvard Medical School, Boston, MA, USA
}

\section{Question 1: Why Do You See Breast Cancer Becoming an Increasing Challenge in Low-Resource Countries?}

Assefa: There are several reasons and challenges in breast cancer care in low-resource countries. The main reason is late presentation of patients. This is due to a lack of awareness about breast cancer signs and symptoms, particularly in the rural setting. Most people associate cancer with pain and visit a health facility when they feel breast pain. On the other hand, they don't seek medical advice despite noting breast lumps for months.

Also, there is a lack of infrastructure/resources for screening and early detection, limited awareness by health professionals resulting in diagnostic delay and a lack of treatment facilities to give treatment on time for patients coming early - this includes access to surgery, chemotherapy and radiotherapy. Also there are no patient support groups (cancer survivors) to teach general public and patients about chances to survive. In our setting I see patients using traditional medicine and seeking religious solutions like using holy water which may delay diagnosis and treatment.

McCormack: Breast cancer is the first or second cause of cancer death in women in almost all countries worldwide. In lower-resource settings, breast cancer needs par- ticular attention because its incidence rates are rising largely due to fertility transitions, whilst the absolute number of women newly diagnosed with this cancer is increasing at an even greater rate amidst the ageing of populations. Thus, for the increasing number of women diagnosed with breast cancer each year, i.e., beyond the 2.1 million women diagnosed globally in 2018 , health systems need to ensure early diagnosis and treatment for women with breast cancer in order to avert what will otherwise be a high mortality toll from this treatable cancer (627,000 deaths in 2018).

Cubasch: The first part of the problem concerns the changing demographics of these countries. We are dealing with generally growing and aging populations. Historically low breast cancer incidence rates in sub-Saharan Africa are increasing due to a westernized lifestyle. In addition, life expectancy is steadily increasing due to HIV being turned into a controllable, chronic disease due to a successful ART roll out. We now need to brace for a secondary epidemic of cancer and other common NCD.

On the other hand, cancer outcomes are much worse than in well-resourced countries; existing health care structures are dysfunctional and there is a lack the resources to deal with the necessary complex multimodality treatment for cancer. The gap between the needs and 
reality is widening. Our patients will have better access to global media channels and be better informed, while health systems will struggle to deliver appropriate care.

Jemal: Breast cancer is increasingly becoming a major public health challenge in low-resource countries for two reasons. First, the burden of the disease is increasing because of increases in the prevalence of known risk factors such as a late child-bearing age, having fewer children per woman, overweight and obesity, and physical inactivity, as well as aging and growth of the population (women) and a shorter duration of breast feeding. Second, the healthcare system is less developed and the healthcare workforce is lacing for timely diagnosis and treatment of breast cancer patients. In most sub-Saharan countries, for example, breast cancer patients are treated without assessing their estrogen-receptor status because of a lack of immunohistochemistry and most surgeries are performed by general surgeons instead of breast surgeons.

Pace: As populations age, and with declining burdens of infectious diseases, we are seeing more and more diseases of aging such as breast cancer. The age-specific breast cancer incidence is also rising, likely because of shifts in reproductive risk factors. We know that, in lowincome countries, women are typically diagnosed late and with stage III or IV disease, which is a major challenge.

\section{Question 2: What Are Important Factors Leading to Disparities in Outcomes Compared to High-Resource Settings?}

Assefa: In low-income countries the double burden of disease, communicable and non-communicable, stretches health system resources. As a result, governments and policy makers are forced to allocate most of the resources for preventive and primary care. Cancer screening and treatment are relatively complex, requiring a tertiary facility with expert trained health professional (radiologist, pathologist, surgeon, oncologist, radiotherapist, and medical physicist, etc., to mention some) and expensive equipment.

McCormack: Low breast cancer survival in lower-resource settings, in comparison to high-resource settings, arises from the accumulation of disparities occurring along the entire journey of women with breast cancer. This journey commences during the many months, sometimes over 1 year, prior to the definitive histological diagnosis and continues during the treatment period.

Cubasch: First of all, in the South African setting we found that it is not due to a more aggressive cancer biology.

Breast Cancer in Low-Resource Countries
However, disparities affect the entire journey of a patient. In the absence of any population screening, the patient usually detects a lump, too often at late stage of disease. Then there is a first contact with the health system, multiple visits and referrals often leading to a delayed diagnosis, further delays with the initiation of chemotherapy, surgery and radiotherapy, non-availability of drugs, and long queues at every step. Many of our patients struggle anyway with hardships of life, money for transport, and concomitant family responsibilities. In other parts of sub-Saharan Africa, patients have to pay for their own diagnostic tests and treatments, which are unaffordable, leading to a nihilistic attitude to cancer.

We found that stage on presentation is not only an important predictor of outcome but it is also a useful process marker that offers itself as a target for possible, affordable interventions.

Jemal: I think major factors that contribute to the breast cancer disparity in low-resource countries compared to high-resource countries include a lack of healthcare facilities, shortage of healthcare workforce, and a lack of treatments.

Pace: There are a lot of factors that contribute to these disparities. Poverty and limited access to primary care services and high-quality breast health services is one factor that contributes to diagnostic delays. Limited access to timely, high-quality diagnostic services and affordable treatment is a major factor. Patients often bear a disproportionate burden of cancer treatment costs. Cancer treatment facilities are often located far from where women live, so that obtaining and completing treatment can be really challenging and expensive. A lack of awareness, thinking that breast cancer is a death sentence, and stigma about cancer and breast cancer in particular may also be a barrier to women accessing care in some settings.

\section{Question 3: How Can Breast Health Be Improved in Low-Income Countries?}

Assefa: Breast cancer care can be improved by increasing public and health professional awareness and by increasing access to the public. This can be achieved by short-term trainings and decentralization of the service in areas with a limited number of tertiary hospitals.

McCormack: The critical factors needing tackling to improve outcomes for women with breast cancer are, in the chronology of the journey with breast cancer, establishing health systems which ensure that women, educated in breast awareness, have access to early initial investigation of breast symptoms and to an accelerated referral 
to diagnosis within 2-3 months of her initial symptom recognition. Such health systems need to support women, along journeys beset by financial, logistical, psychologi$\mathrm{cal}$, transport, and spatiotemporal associated challenges, to her diagnosis and through complete treatment courses.

Cubasch: The situational analysis will differ from country to country and there is no magic bullet. South Africa is considered an upper-middle income country, but due to the high index of social inequality we share many problems with low-income countries.

It is an important starting point to collect good-quality cancer data - thus giving shape to the beast (i.e., a baseline analysis for future trend evaluations, and assessment of the success or otherwise of interventions aimed at improving survival). The South African National Cancer Registry is based on pathology reports from the public and private sector. There are also a few patchy institutional databases (such as academic hospital cohort database registries) and there are a few collaborative research projects pooling those data.

It is a clinical imperative in poorly resourced settings to come up with inexpensive solutions for earlier-stage diagnosis and treatment. Examples are widespread implementation of clinical breast examination, use of diagnostic ultrasound and fine-needle aspiration, hormonal treatment, and hypo-fractionated DXT regimens, to name just a few.

High-volume activities in dedicated centers can contribute to an economy of scale and prevent wastage of scarce health resources.

Jemal: There are several measures that can be taken to improve breast health in low-resource countries. The major reason for poor breast outcomes in low-resource countries is late diagnosis of the disease. This problem could be mitigated by increasing awareness about the importance of early detection of breast cancer among the general population as well as among the healthcare providers. In addition, local governments should develop public health policies and recommendations to promote heathy behaviors such as maintaining a healthy bodyweight, staying physically active, and breast feeding. Strengthening the north-south partnership between academia and research institutes to share best practice could also help improve breast cancer outcomes in low-resource countries.

Pace: I think that figuring out how to integrate breast health and breast cancer early detection (focusing first on the care of women with breast symptoms) into routine health services, and establishing referral and navigation systems to support women during evaluation and treatment, are critical steps needed to facilitate earlier diagnoses.

\section{Question 4: Which Specific Interventions Have Been Shown to Be Successful to Implement Breast Cancer Care in the Public Health Care Systems?}

Assefa: Each country should include short- and longterm intervention in its national cancer control program to tackle breast cancer using cost effective measures. The main treatment of breast cancer in low-resource settings where radiotherapy is not available is mastectomy. This can be done at district hospitals after training general surgeons. Since more than $60 \%$ of breast cancers are hormone receptor positive, hormonal therapy can be given even without testing and this can greatly improve outcomes for most patients. This is also possible in lowerlevel hospitals. At the same time, at least first-generation adjuvant chemotherapy when available can be given after short training in district hospitals. All of these are cost effective measures which improve outcomes and can easily be incorporated into the existing health system.

McCormack: Successful strategies to improve breast cancer outcomes, i.e., ultimately averting deaths through improving intermediate end-points along the entire breast cancer journey, will inherently be setting specific, tailored to cultures, sociodemographic profiles, and the health system. Nevertheless, promising interventions include training of health workers in breast health and in clinical breast examinations and the presence of reliable breast clinics, accompanied by good-quality timely pathology and treatment, linked within a clear referral system to first-line care providers. On the treatment side, more studies are needed on how to improve percentages of women commencing and completing recommended therapeutic courses within a timely fashion.

Cubasch: In South Africa the public sector caters for $85 \%$ of the population.

A decisive step for breast care was the establishment of highly visible, dedicated breast services, often driven by academic surgeons who subspecialize in breasts and work in the context of a multidisciplinary team.

In our own unit we focused on a strict open-door policy and eliminated all unnecessary hurdles and waiting lists.

Real-time electronic data collection opened the way to research projects and international collaborations.

In an extensive process of consultation, the government has embarked on drafting a breast cancer control policy; however, a widespread practical implementation is still outstanding.

Following the experience from the struggle to access HIV treatment, a critical role has to be played by NGO and lobby groups. The SA Cancer Alliance is a covering body that allows the civil society to speak with one voice and to be the advocate for our patients. 
Pace: In Rwanda, we have learned several lessons about implementing breast cancer care. Rwanda's first public cancer facility, i.e., the Butaro Cancer Center of Excellence, has shown that non-oncologists can safely and effectively deliver cancer care, with intensive training, ongoing support and mentorship, and context-specific protocols. Addressing patients' socioeconomic needs is also really important. Rwanda's national health insurance is important for helping patients access regular primary care and referral services, but eligible patients at Butaro also receive additional support such as transport fees to help them continue receiving care. We also offer breast cancer support groups which allow women to build support networks, share experiences, and learn about their disease and treatment, and there is a growing network of breast cancer survivors in the country who support each other and advocate for improved access to care.

\section{Participants}

Dr. Mathewos Assefa

Radiotherapy Center

School of Medicine, College of Health Sciences, Addis Ababa

University

Tikur Anbessa Specialized Hospital

PO Box 9086, Addis Ababa (Ethiopia)

E-Mail mathewosassefa80@hotmail.com
Dr. Valerie McCormack

Section of Environment and Radiation

International Agency for Research on Cancer

150 Cours Albert Thomas, 69372 Lyon Cedex 08 (France)

E-Mail McCormackV@iarc.fr

Dr. Herbert Cubasch

Department of Surgery, Faculty of Health Sciences

University of the Witwatersrand

7 York Rd., Parktown, Johannesburg 2193 (South Africa) and

Batho Pele Breast Unit

Chris Hani Baragwanath Academic Hospital

26 Chris Hani Rd., Diepkloof, Johannesburg 1864 (South Africa)

E-Mail hcubasch@gmail.com

Dr. Ahmedin Jemal, DVM, PhD

Scientific Vice President, Surveillance and Health Services Research

American Cancer Society, Inc.

250 Williams St., Atlanta, GA 30303 (USA)

E-Mail ahmedin.jemal@cancer.org

Dr. Lydia E. Pace

Brigham and Women's Hospital

Department of Medicine

75 Francis St., Boston, MA 02115 (USA)

E-Mail lpace@bwh.harvard.edu

\section{Disclosure Statement}

The participants have no conflict of interests to report. 